\title{
Diagnosis of primary ciliary dyskinesia: searching for a gold standard
}

\author{
Jane S. Lucas ${ }^{1,2}$ and Margaret W. Leigh ${ }^{3,4}$ \\ Affiliations: ${ }^{1}$ Primary Ciliary Dyskinesia Centre, University Hospital Southampton NHS Foundation Trust, \\ Southampton, UK. ${ }^{2}$ Academic Unit of Clinical and Experimental Sciences, Faculty of Medicine, University of \\ Southampton, Southampton, UK. ${ }^{3}$ Dept of Pediatrics, University of North Carolina School of Medicine, Chapel \\ Hill, NC, USA. ${ }^{4}$ Both authors contributed equally to this manuscript.
}

\section{Correspondence:}

Jane S. Lucas, Primary Ciliary Dyskinesia Centre, Clinical and Experimental Sciences Academic Unit (Mail Point 803), University of Southampton Faculty of Medicine, University Hospital Southampton NHS Foundation Trust, Tremona Road, Southampton, S016 6YD, UK. E-mail: jlucas1asoton.ac.uk

Margaret W. Leigh, Dept of Pediatrics, University of North Carolina School of Medicine, Chapel Hill, NC, USA. E-mail: margaret_leighamed.unc.edu

0 @ERSpublications

Despite advances, standardised methods to diagnose primary ciliary dyskinesia are still needed http://ow.ly/CMYsf

Significant advances have been made in the diagnosis of patients with primary ciliary dyskinesia (PCD) including centralised services [1-3], European consensus guidelines [4], systematic, protocol-directed diagnostic testing in a national service in the UK [2] and in a PCD research consortium in North America [1], and evidence for standardisation of nasal nitric oxide testing as a test for PCD [5]. Moreover, there have been striking advances in discovery of PCD genes and multigene panels in Europe and North America. Despite these developments there remains no "gold standard" single, diagnostic test for PCD. Thus, the diagnosis usually requires a number of technically demanding, sophisticated investigations that are not available in many centres or countries.

The manuscript by RAIDT et al. [6], in this edition of the European Respiratory Journal, describes a large cohort of patients with defined mutations in multiple PCD genes and the associated abnormalities of ciliary pattern observed by high-speed video-microscopy analysis (HVMA), using freshly obtained nasal epithelium. Their findings are consistent with prior reports of altered ciliary waveforms associated with mutations in specific PCD genes. For example, different mutations in DNAH5 all resulted in minimal residual beating which was disorganised. They also confirmed a previous report [7] suggesting that different mutations in the same gene can be associated with different ciliary patterns, with mutations in DNAH11 resulting in stiff hyperkinetic beating in some families while another patient had mostly static cilia [6]. A limitation of the study was small numbers of patients with individual gene defects. Furthermore, it is not clear whether the definition of ciliary beat abnormality was established before or after other diagnostic tests and identification of gene mutations. A prospective, multicentre study will be required to include sufficient families with this rare disease (as well as healthy and disease controls) to fully understand the associations between gene mutations and ciliary function. This study demonstrates the value of genetic testing in PCD and it is hoped that the study will contribute to the availability of genotyping in countries where it is not yet available.

Received: Sept 242014 | Accepted after revision: Oct 112014

Support statement: J.S. Lucas is supported by the National PCD Diagnostic Service at UHS commissioned and funded by NHS England; her research is supported by NIHR Southampton Respiratory Biomedical Research Unit and NIHR Wellcome Trust Clinical Research Facility, Southampton, UK. M. Leigh is supported by NIH 5 U54 HL09640958 funded by the Office of Rare Diseases Research (ORDR, NCATS), and administered by NIH Heart, Lung and Blood Institute (Bethesda, MD, USA). Both J.S. Lucas and M. Leigh are supported by EU-FP7 BESTCILIA 305404.

Conflict of interest: Disclosures can be found alongside the online version of this article at erj.ersjournals.com

Copyright @ERS 2014 


\section{Evolution of diagnostic testing}

Throughout the history of PCD, understanding of the underlying pathophysiology and advances in technology has driven the development of diagnostic tests. Bronchiectasis associated with situs inversus was first reported by Siewart in 1904, followed by Kartagener's description of the triad of situs inversus, bronchiectasis and sinusitis in 1933. It was not until 1976 that ciliary defects were identified as the causative link, when immotile cilia with absent dynein arms were described in the cilia of three men with sinopulmonary disease and immotile sperm [8]. Using light microscopy to evaluate ciliary beat and transmission electron microscopy (TEM) to examine ciliary ultrastructure, other patients with chronic sinopulmonary disease were diagnosed with "immotile cilia syndrome" [8]. Approximately 50\% of the patients with immotile cilia syndrome had situs inversus totalis, leading AfZELIUs [8] to speculate that cilia were important for directing laterality. Subsequent studies identified patients with sinopulmonary disease who had motile, but dyskinetic ciliary beat leading to the term "primary ciliary dyskinesia" [9]. Subsequent TEM studies in the 1980's identified several different ultrastructural defects in patients with PCD, including absence of outer dynein arms, absence of outer and inner dynein arms, and absence of the central pair with transposition of a peripheral doublet into the central position. In the 1990's, PCD patients were noted to have very low levels of nitric oxide [10] and subsequent studies confirmed this finding, leading to incorporation of nasal nitric oxide (nNO) testing into the diagnostic evaluation for PCD [5, 11] even though the underlying mechanism remains elusive [12]. Understanding the genetic basis of PCD has increased exponentially since mutations in DNAI1 were reported to cause PCD in 1999 [13]. To date, mutations in over 30 PCD-associated genes have been identified, accounting for $>60 \%$ of PCD cases [1]. With over 250 proteins in the cilium, and additional proteins involved in the cytoplasmic pre-assembly and/ or transport of dyneins, the number of genes still to be identified is potentially very large. This extensive genetic heterogeneity makes recent developments in next generation sequencing approaches attractive both for gene discovery research and for use in the clinical setting, as they offer parallel sequencing of multiple candidate genes or of the entire exome.

Over the past 15 years, advances in technology and discovery of PCD genes have enhanced our understanding of ciliary pathophysiology. For example, attachment of a high-speed video camera to a light microscope allowed an experienced cilia scientist to evaluate ciliary wave form at slow speed and identify more subtle changes in ciliary beat that cannot be appreciated by simple light microscopy. Studies in 2003 showed that certain patterns of ciliary dyskinesia apparent on HVMA are associated with specific ultrastructural defects [14]. A key aspect of characterising newly defined PCD genes has included careful definition of clinical phenotype, ciliary function by HVMA, ultrastructural changes on TEM and nNO values in patients with the mutated gene. For most genes, defects in ciliary beat are consistently associated with the ultrastructural defect as shown in prior studies. However, some of the newly defined genes are associated with novel defects in ciliary waveform. For example, patients with DNAH11 mutations have normal ciliary ultrastructure but stiff, hyperkinetic $[6,15]$ or static $[6,7]$ ciliary beat and low nNO. In addition, careful definition of ciliary defects associated with recently identified genes have defined novel "hallmark" ultrastructural defects such as absence of inner dynein arms combined with central apparatus defects and microtubular disorganisation in patients with mutations in CCDC39 and CCDC40 genes, which are associated with an altered ciliary beat ranging from stiff beat, with low amplitude and frequency, to completely immotile [16]. For most of the over 30 PCD genes described to date, nNO values have been very low, with the exception of RSPH1, for which several patients have had normal or non-diagnostic nNO values [17].

Despite these advances, the diagnosis of PCD can be challenging. No single diagnostic test is perfect for diagnosing PCD and a combination of tests is required. Each test has limitations and requires specialised expertise. As with any diagnosis, definition of a clinical phenotype that provides robust a priori prediction of PCD is crucial. Ciliary motility is abnormal in most patients with PCD; however, subtle defects are increasingly recognised and require analyses by scientists with substantial experience with normal and abnormal cilia for accuracy. RAIDT et al. [6] discuss some of the issues that they encountered using HVMA including obtaining adequate visualisation for analysis and choice of ambient temperature. These issues are fundamental for all diagnostic centres and warrant international discussion and consensus. Moreover, abnormal ciliary function can be secondary to infectious or inflammatory damage to the native tissue before biopsy or to damage during biopsy or mishandling of the sample prior to recording of HVMA. Repeat testing or reanalysis following culture at the air-liquid interface (ALI) [18] or under submerged conditions [19] has, therefore, been recommended [4] to confirm primary rather than secondary defects. Most defects of ciliary ultrastructure defined in adequate numbers of cilia with good preparation and sampling of images have $100 \%$ specificity; however, analysis of cilia by TEM will miss the $30 \%$ of patients who do not have "hallmark" ultrastructural defects [20]. Some ultrastructural defects (e.g. those associated with RSPH9 and RSPH4A [21] or RSPH1 [17, 22]) are subtle and can be difficult to detect by all but those with the most experience in electron microscopy interpretation. In addition, electron microscopy reviewers need extensive 
experience with the range of secondary defects associated with unhealthy cells as well as the range of defects associated with PCD. Measurement of $\mathrm{nNO}$ by a trained technician is relatively straightforward [5], but rare patients with PCD may have nNO levels in the normal range [11,17]; and patients with other causes of bronchiectasis, particularly cystic fibrosis, may have levels in the diagnostic range $\left(<77 \mathrm{~nL} \cdot \mathrm{min}^{-1}\right)$ although not in the very low range $\left(<30 \mathrm{~nL} \cdot \mathrm{min}^{-1}\right)$ seen in the vast majority of PCD patients $[5,11]$. Because nNO can be falsely low during an acute upper respiratory infection, repeat testing may be needed. nNO is frequently low in very young healthy children $(<2$ years of age), making it non-discriminatory in these patients. At present, genetic analysis for $>30$ PCD genes fails to detect approximately $30 \%$ of patients [1], but this figure continues to decrease as new PCD genes are identified. Expertise is essential to interpretation of genetic testing; the authors are aware of patients who have been misdiagnosed by interpretation-error of gene variants of unknown significance identified by commercial genetic testing. Additional tests that may be helpful when other diagnostic tests are inconclusive include: immunofluorescence labelling of ciliary proteins [23] and in vivo assessment of mucociliary clearance of radiolabelled aerosol [24, 25].

Interpretation of test results is not currently guided by international consensus. Identification of "hallmark" defects on TEM or biallelic disease-causing mutations in a PCD gene is straightforward; similarly, identification of a "hallmark" HVMA finding (e.g. "static" cilia or very stiff, dyskinetic cilia) on at least two separate occasions when clinically stable provides strong evidence for PCD. However, not all PCD patients meet these criteria. As observed in patients with DNAH11 mutations, some patients may have biallelic mutations in a PCD gene but more subtle changes in HVMA only detected by experienced scientists using high resolution equipment, and normal ultrastructure. Historically, the term "atypical PCD” was inappropriately used for these patients even though they have "typical" clinical manifestations of PCD. An ERS Task Force (ERS TF-2014-04) is currently developing an evidence-based practice guideline for the diagnosis of PCD; one of their remits is to develop an algorithm for how tests results can be interpreted to decide whether a patient's PCD diagnosis is positive, negative or inconclusive.

Since HVMA, nNO, TEM and genetic testing require expensive equipment, infrastructure and expert scientists PCD diagnostic services have generally developed in centres with established PCD and ciliary research programs that have expertise in some, but not all, of these testing modalities. Therefore, historical development of diagnostic services has led to variations in the combination of tests available in different countries. Importantly, there is no clear "gold standard" test and no consensus on the conduct of either individual investigations or the combination of tests.

We present the experiences of centres in England, where genetic testing is not clinically available, and from USA, where HVMA is not routine.

\section{Diagnostic testing in England (Jane Lucas)}

Three centres that were already performing diagnostic testing on a research basis were commissioned to provide a service for adults and children within the infrastructure of the National Health Service $[2,3]$. Hospitals in Southampton, London and Leicester work collaboratively with shared protocols, competencybased training of scientists, difficult case meetings and cross-centre audits. A network of referral hospitals takes samples from patients who are geographically distant from the centres. These samples are sent by courier to the three centres preventing unnecessary travel by patients. The centres use a combination of techniques including nNO, HVMA, TEM and culture at the ALI to contribute to a diagnosis of PCD, but genotyping is only conducted on a research basis dependent on grant funding. The small team of highly specialised scientists at each centre, analysing a high throughput of PCD-diagnostic samples provides a depth of experience which is particularly important for defects with subtle defects of function or ultrastructure.

The opinion in England, as in much of Europe, is that HVMA is central to PCD diagnostics. In expert hands HVMA will detect almost all abnormalities of ciliary beat pattern; to confirm the defects are consistent the HVMA testing is repeated after 3-6 months or reanalysed following culture at the ALI [18]. TEM provides further diagnostic information regarding the underlying defect, but will fail to detect $30 \%$ of cases. For some years the service has been aware of a small percentage of patients with a typical clinical history of PCD, and consistently "equivocal" ciliary beat pattern, always subtly abnormal on repeat testing. The National PCD Service would welcome genetic testing as an additional diagnostic test if the funding could be secured, now that genetic mutations have been identified which might clarify these difficult cases.

\section{Diagnostic testing in North America (Margaret Leigh)}

Eight centres across North America are involved in the Genetic Disorders of Mucociliary Clearance Consortium within the Rare Diseases Clinical Research Network funded by the US National Institutes of Health. This consortium developed standardised protocols for diagnostic testing, including systematic assessment of clinical features to define the clinical phenotype predictive of PCD, nNO testing, nasal ciliary 
biopsy for electron microscopy (with shipment of samples in fixative to a central centre at the University of North Carolina for processing, sectioning and systematic capturing of TEM images for analysis by three independent reviewers) and obtaining DNA for research genetic testing. Through systematic assessment of clinical features, we have established clinical criteria for the PCD phenotype. Through standardisation of $\mathrm{nNO}$ and TEM for a large number of individuals evaluated at these North American centres (complemented by research genetic testing), we have been able to establish and validate a clear cut-off value for nNO [5] and a consistent quality of electron micrographs for interpretation, thereby, limiting subjectivity. These approaches have been critical to our efforts to identify novel PCD genes in collaboration with our international colleagues. HVMA is limited to the one site that has appropriate equipment and expertise. In our hands, HVMA provides useful information about ciliary function, but is limited by subjectivity in analysis and nonspecific changes that can confound interpretation. Some samples have regional variation in ciliary beat pattern (some areas are normal but others have a stiff beat pattern). Therefore, we concluded that HVMA needed further standardisation of methodology and interpretation of ciliary beat pattern as well as assessment of inter-reader variability before it could be used as a primary diagnostic test. Through research genetic testing, we have been able to define biallelic mutations in a PCD gene in over 70\% of our PCD patients and anticipate that this percentage will increase rapidly, based on the rate of discovery of new PCD genes in the past 3 years. Our goal is to use clinical phenotyping and nNO testing to identify patients likely to have PCD followed by genetic testing to confirm the diagnosis, reserving HVMA and TEM for cases that are not identified by PCD multigene panel testing.

\section{Summary}

Historically, diagnosis of PCD has advanced with increased knowledge of pathophysiology and advancing technology. Recent identification of PCD-causing genes provides a more rigorous platform for testing "advancing technologies", as seen in the study by RAIDT et al. [6]. Currently available tests for PCD include nNO, HVMA, TEM and PCD genetic testing. At present, no one test outperforms all the others and each is undergoing different stages of standardisation for clinical application. The study by RAIDT et al. [6] provides summary information about the range of ciliary beat patterns associated with specific genetic mutations and identifies some of the limitations of this approach. With advances in PCD gene discovery, we are entering a new phase in diagnostic testing where PCD gene analysis will be able to identify an increasing proportion of PCD patients, even those who are not easily identified by HVMA and TEM.

\section{Acknowledgements}

J.S. Lucas and M. Leigh are members of European Respiratory Society (ERS) Task Force to develop a practice guideline for diagnosis of PCD (ERS TF-2014-04); this editorial does not represent the views of the ERS Task Force.

\section{References}

1 Knowles MR, Daniels LA, Davis SD, et al. Primary ciliary dyskinesia. Recent advances in diagnostics, genetics, and characterization of clinical disease. Am J Respir Crit Care Med 2013; 188: 913-922.

2 Lucas JS, Burgess A, Mitchison HM, et al. Diagnosis and management of primary ciliary dyskinesia. Arch Dis Child 2014; 99: 850-856.

O'Callaghan C, Chilvers M, Hogg C, et al. Diagnosing primary ciliary dyskinesia. Thorax 2007; 62: 656-657.

4 Barbato A, Frischer T, Kuehni CE, et al. Primary ciliary dyskinesia: a consensus statement on diagnostic and treatment approaches in children. Eur Respir J 2009; 34: 1264-1276.

5 Leigh MW, Hazucha MJ, Chawla KK, et al. Standardizing nasal nitric oxide measurement as a test for primary ciliary dyskinesia. Ann Am Thorac Soc 2013; 10: 574-581.

6 Raidt J, Wallmeier J, Hjeij R, et al. Ciliary beat pattern and frequency in genetic variants of primary ciliary dyskinesia. Eur Respir J 2014; 44: 1579-1588.

7 Lucas JS, Adam EC, Goggin PM, et al. Static respiratory cilia associated with mutations in Dnahc11/DNAH11: a mouse model of PCD. Hum Mutat 2012; 33: 495-503.

Afzelius BA. A human syndrome caused by immotile cilia. Science 1976; 193: 317-319.

Sleigh MA. Primary ciliary dyskinesia. Lancet 1981; 2: 476.

10 Lundberg JO. Nitric oxide and the paranasal sinuses. Anat Rec (Hoboken) 2008; 291: 1479-1484.

11 Collins SA, Gove K, Walker W, et al. Nasal nitric oxide screening for primary ciliary dyskinesia: systematic review and meta-analysis. Eur Respir J 2014; 44: 1589-1599.

12 Walker WT, Jackson CL, Lackie PM, et al. Nitric oxide in primary ciliary dyskinesia. Eur Respir J 2012; 40: $1024-1032$.

13 Pennarun G, Escudier E, Chapelin C, et al. Loss-of-function mutations in a human gene related to Chlamydomonas reinhardtii dynein IC78 result in primary ciliary dyskinesia. Am J Hum Genet 1999; 65: 1508-1519.

14 Chilvers MA, Rutman A, O'Callaghan C. Ciliary beat pattern is associated with specific ultrastructural defects in primary ciliary dyskinesia. J Allergy Clin Immunol 2003; 112: 518-524.

15 Schwabe GC, Hoffmann K, Loges NT, et al. Primary ciliary dyskinesia associated with normal axoneme ultrastructure is caused by DNAH11 mutations. Hum Mutat 2008; 29: 289-298.

16 Antony D, Becker-Heck A, Zariwala MA, et al. Mutations in CCDC39 and CCDC40 are the major cause of primary ciliary dyskinesia with axonemal disorganization and absent inner dynein arms. Hum Mutat 2013; 34: 462-472.

17 Knowles MR, Ostrowski LE, Leigh MW, et al. Mutations in RSPH1 cause primary ciliary dyskinesia with a unique clinical and ciliary phenotype. Am J Respir Crit Care Med 2014; 189: 707-717. 
18 Hirst RA, Jackson CL, Coles JL, et al. Culture of primary ciliary dyskinesia epithelial cells at air-liquid interface can alter ciliary phenotype but remains a robust and informative diagnostic aid. PLoS One 2014; 9: e89675.

19 Jorissen M, Willems T, Van der Schueren B. Ciliary function analysis for the diagnosis of primary ciliary dyskinesia: advantages of ciliogenesis in culture. Acta Otolaryngol 2000; 120: 291-295.

20 Boon M, Smits A, Cuppens $\mathrm{H}$, et al. Primary ciliary dyskinesia: critical evaluation of clinical symptoms and diagnosis in patients with normal and abnormal ultrastructure. Orphanet J Rare Dis 2014; 9: 11.

21 Castleman VH, Romio L, Chodhari R, et al. Mutations in radial spoke head protein genes RSPH9 and RSPH4A cause primary ciliary dyskinesia with central-microtubular-pair abnormalities. Am J Hum Genet 2009; 84: 197-209.

22 Kott E, Legendre M, Copin B, et al. Loss-of-function mutations in RSPH1 cause primary ciliary dyskinesia with central-complex and radial-spoke defects. Am J Hum Genet 2013; 93: 561-570.

23 Omran H, Loges NT. Immunofluorescence staining of ciliated respiratory epithelial cells. Methods Cell Biol 2009; 91: 123-133.

24 Marthin JK, Mortensen J, Pressler T, et al. Pulmonary radioaerosol mucociliary clearance in diagnosis of primary ciliary dyskinesia. Chest 2007; 132: 966-976.

25 Walker WT, Young A, Bennett M, et al. Pulmonary radioaerosol mucociliary clearance in primary ciliary dyskinesia. Eur Respir J 2014; 44: 533-535. 